\title{
On the Korovkin-type approximation of set-valued continuous functions
}

\author{
MiCHELE CAMPITI*
}

\begin{abstract}
This paper is devoted to some Korovkin approximation results in cones of Hausdorff continuous setvalued functions and in spaces of vector-valued functions. Some classical results are exposed in order to give a more complete treatment of the subject. New contributions are concerned both with the general theory than in particular with the so-called convexity monotone operators, which are considered in cones of set-valued function and also in spaces of vector-valued functions.
\end{abstract}

Keywords: Korovkin approximation, approximation of vector-valued functions, approximation of set-valued functions.

2020 Mathematics Subject Classification: 41A65, 41A36, 41A25, 41A63.

Dedicated to Professor Francesco Altomare, on occasion of his 70th birthday, with esteem and friendship.

\section{INTRODUCTION AND NOTATION}

This paper is devoted to some aspects of the Korovkin approximation theory which has been deeply studied in the last decades and is concerned with the approximation of operators between cones of Hausdorff continuous set-valued functions. It is partially a survey of preceding results but contains also some new results which completes the analysis carried out in the following sections. My attention to this topic was pointed out and strongly encouraged by Professor Francesco Altomare throughout many discussions on the pioneristic work by Keimel and Roth [13,14]. Some important consequences are also concerned with Korovkin approximation theory in spaces of continuous real functions and also in this case we shall give some new contributions.

The paper is organized as follows. Section 2 is devoted to classical results together with some new results strictly connected to the classical theory. In Section 3, we consider a particular class of linear operators, the so-called convexity monotone operators, which allow to point out how the analysis of set-valued functions is strictly connected with that one of vector-valued functions. We shall state some consequences of the results in Section 4 and in the same section we shall state some new results concerning the Korovkin approximation of vector-valued functions which generalize some recent results obtained in the space $C([0,1])$. We can also state further developments concerning Korovkin approximation of set-valued functions.

In the following sections, we shall denote by $\mathbf{B}$ the closed unit ball with center 0 in $\mathbb{R}^{d}(d \geq 1)$ and by $e_{1}, \ldots, e_{d}$ the canonical base of $\mathbb{R}^{d}$. Moreover, $\mathcal{K}\left(\mathbb{R}^{d}\right)$ will denote the cone of all nonempty compact convex subsets of $\mathbb{R}^{d}$ endowed with the natural addition and multiplication by

Received: 17.01.2021; Accepted: 28.01.2021; Published Online: 01.03.2021

*Corresponding author: Michele Campiti; michele.campiti@unisalento.it

DOI: $10.33205 / \mathrm{cma} .863145$ 
positive scalars. In $\mathcal{K}\left(\mathbb{R}^{d}\right)$, we consider the Hausdorff distance

$$
d_{H}(A, B):=\inf \{\varepsilon>0 \mid A \subset B+\varepsilon \cdot \mathbf{B}, B \subset A+\varepsilon \cdot \mathbf{B}\} .
$$

If $X$ is an Hausdorff topological space, we shall deal with the cone $C\left(X, \mathcal{K}\left(\mathbb{R}^{d}\right)\right)$ of all Hausdorff continuous set-valued functions; hence, $f \in C\left(X, \mathcal{K}\left(\mathbb{R}^{d}\right)\right)$ if and only if for every $x_{0} \in X$ and $\varepsilon>0$, there exists a neighborhood $U$ of $x_{0}$ such that $f(x) \subset f\left(x_{0}\right)+\varepsilon \cdot \mathbf{B}$ and $f\left(x_{0}\right) \subset f(x)+\varepsilon \cdot \mathbf{B}$ whenever $x \in U$. The space $C\left(X, \mathcal{K}\left(\mathbb{R}^{d}\right)\right)$ is naturally ordered by inclusion, that is

$$
f \leq g \Leftrightarrow \forall x \in X: f(x) \subset g(x)
$$

whenever $f, g \in C\left(X, \mathcal{K}\left(\mathbb{R}^{d}\right)\right)$. An operator $T: C\left(X, \mathcal{K}\left(\mathbb{R}^{d}\right)\right) \rightarrow C\left(X, \mathcal{K}\left(\mathbb{R}^{d}\right)\right)$ is called linear if it preserves addition and multiplication by positive scalars and is called monotone if it preserves inclusions. If $\varphi \in C\left(X, \mathbb{R}^{d}\right)$, it will be useful to denote by $\{\varphi\}$ the set-valued function (in $\left.C\left(X, \mathcal{K}\left(\mathbb{R}^{d}\right)\right)\right)$ defined by setting $\{\varphi\}(x)=\{\varphi(x)\}$ for every $x \in X$. Moreover, if $\varphi_{1}, \ldots, \varphi_{n} \in$ $C\left(X, \mathbb{R}^{d}\right)$ are set-valued functions on $X$, we can consider the set-valued function $\operatorname{co}\left(\varphi_{1}, \ldots, \varphi_{n}\right)$ defined by

$$
\operatorname{co}\left(\varphi_{1}, \ldots, \varphi_{n}\right)(x)=\operatorname{co}\left(\varphi_{1}(x), \ldots, \varphi_{n}(x)\right)
$$

for every $x \in X$, where $\operatorname{co}\left(\varphi_{1}(x), \ldots, \varphi_{n}(x)\right)$ denotes the convex hull of $\varphi_{1}(x), \ldots, \varphi_{n}(x)$. Moreover, if $f \in C\left(X, \mathcal{K}\left(\mathbb{R}^{d}\right)\right)$, we shall denote by $\operatorname{Sel}(f)$ the convex subset of $C\left(X, \mathcal{K}\left(\mathbb{R}^{d}\right)\right)$ consisting of all continuous selections of $f$. It is well-known that (see e.g. [13, Lemma 4.1. and Theorem 3.2] or [4, Proposition 1.1])

$$
f=\bigcup_{\varphi \in \operatorname{Sel}(f)}\{\varphi\}
$$

for every $f \in C\left(X, \mathcal{K}\left(\mathbb{R}^{d}\right)\right)$, that is $f(x)=\bigcup_{\varphi \in \operatorname{Sel}(f)}\{\varphi(x)\}$ for every $x \in X$.

\section{SOME CLASSICAL RESULTS}

The starting point is the extension of the classical Korovkin theorem to the setting of Hausdorff continuous set-valued functions. We recall that a subset $M$ of $C(X, \mathbb{R})$ is called a Korovkin system in $C(X, \mathbb{R})$ if whenever $\left(T_{i}\right)_{i \in I}^{\leq}$is an equicontinuous net of positive linear operators from $C(X, \mathbb{R})$ into itself satisfying the condition $\lim _{i \in I}^{\leq} T_{i}(\varphi)=\varphi$ for every $\varphi \in M$, we also have $\lim _{i \in I}^{\leq} T_{i}(\psi)=\psi$ for every $\psi \in C(X, \mathbb{R})$. If the limit operator is not the identity operator but a linear positive operator $T: C(X, \mathbb{R}) \rightarrow C(X, \mathbb{R})$, we shall say that $M$ is called a $T$-Korovkin system (or a Korovkin system for $T$ ) in $C(X, \mathbb{R})$. In the space $C\left(X, \mathcal{K}\left(\mathbb{R}^{d}\right)\right.$ ), we have a similar definition (see $[13,4])$. Namely, let $\mathcal{C}$ be a subcone of $C\left(X, \mathcal{K}\left(\mathbb{R}^{d}\right)\right)$; a subset $H$ of $\mathcal{C}$ is called a Korovkin system in $\mathcal{C}$ if for every equicontinuous net $\left(L_{i}\right)_{i \in I}^{\leq}$of linear monotone operators from $\mathcal{C}$ into itself satisfying $\lim _{i \in I}^{\leq} L_{i}(h)=h$ for every $h \in H$, we also have $\lim _{i \in I}^{\leq} L_{i}(f)=f$ for every $f \in \mathcal{C}$. Also in this case, we shall call $H$ a $L$-Korovkin system (or a Korovkin system for $L)$ in $\mathcal{C}$ in the case where the limit operator is not the identity operator but a continuous linear monotone operator $L: \mathcal{C} \rightarrow \mathcal{C}$. The existence of Korovkin systems in $C\left(X, \mathbb{R}^{d}\right)$ has been widely studied and a complete treatment can be found in [1, 2]. In despite of this, we do not have a complete analysis of Korovkin systems in $C\left(X, \mathcal{K}\left(\mathbb{R}^{d}\right)\right)$ and our aim is to add some contribution to this topic. First of all, an extension of Korovkin systems to the case of set-valued functions was obtained by Keimel and Roth in [13] and can be stated as follows.

Theorem 2.1. ([13, Theorem 3.1]) Let $X$ be a compact Hausdorff topological space and let $M$ be a Korovkin system of positive functions in $C(X, \mathbb{R})$. Then, the subset consisting of all the functions $f \cdot \boldsymbol{B}$ (for every $x \in X: f \cdot \boldsymbol{B}(x):=f(x) \cdot \boldsymbol{B}$ ) and the constant functions $\boldsymbol{B}, e_{1}, \ldots, e_{d}$, is a Korovkin system in $C\left(X, \mathcal{K}\left(\mathbb{R}^{d}\right)\right)$. 
It follows in particular that the functions

$$
x \mapsto \mathbf{B}, \quad x \mapsto x \cdot \mathbf{B}, \quad x \mapsto x^{2} \cdot \mathbf{B}
$$

form a Korovkin system in $C(X, \mathcal{K}(\mathbb{R}))$. A refinement of this result has been obtained in [4] in terms of upper and lower envelopes.

Theorem 2.2. ([4, Theorem 2.2]) Let $X$ be a compact Hausdorff topological space and let $H$ be a subset of $C\left(X, \mathcal{K}\left(\mathbb{R}^{d}\right)\right)$. Assume that there exists a Korovkin system of positive functions $M$ in $C(X, \mathbb{R})$ such that, for every $\varphi \in M, x_{0} \in X$ and $\varepsilon>0$,

$$
\left(\varphi\left(x_{0}\right)+\varepsilon\right) \cdot \boldsymbol{B}=\overline{\bigcup_{f \in H, f \leq(\varphi+\varepsilon) \cdot \boldsymbol{B}} f\left(x_{0}\right)}=\bigcap_{f \in H,(\varphi+\varepsilon) \cdot \boldsymbol{B} \leq f} f\left(x_{0}\right) .
$$

Then, $H$ is a Korovkin system in $C\left(X, \mathcal{K}\left(\mathbb{R}^{d}\right)\right)$.

The assumptions in Theorem 2.1 ensure that for every $\varphi \in M$ and $\varepsilon>0$, the function $(\varphi+\varepsilon) \cdot \mathbf{B} \in H$ and hence the assumptions in Theorem 2.2 are trivially satisfied. Therefore, Theorem 2.2 generalizes Theorem 2.1. A more meaningful extension of the preceding results can be found in [5] in a more general setting and even in the case where the limit operator is not necessarily the identity operator. In this regard, we recall the following result obtained in [5, Theorem 2.4 and Corollary 2.5].

Theorem 2.3. ([5, Theorem 2.4]) Let $X$ be a compact Hausdorff topological space, $\mathcal{C}$ a subcone of $C\left(X, \mathcal{K}\left(\mathbb{R}^{d}\right)\right)$ containing the single-valued functions and $L: \mathcal{C} \rightarrow \mathcal{C}$ a continuous monotone linear operator satisfying the following conditions:

a) For every $\varphi \in C\left(X, \mathbb{R}^{d}\right), L(\{\varphi\})$ is single-valued;

b) For every $f \in \mathcal{C}$ and $x \in X$ :

$$
L(f)(x)=\bigcup_{\varphi \in \operatorname{Sel}(f)} L(\{\varphi\})(x) .
$$

Let $H$ be a subset of $\mathcal{C}$ such that, for every $f \in \mathcal{C}, x_{0} \in X$ and $\varepsilon>0$, there exists $h \in H$ satisfying the following conditions:

$$
f \leq h, \quad L(h)\left(x_{0}\right) \subset L(f)\left(x_{0}\right)+\varepsilon \cdot \boldsymbol{B} .
$$

Then, $H$ is a Korovkin system for $L$ in $\mathcal{C}$.

As observed in [5, Remark 2.6], if $H$ contains the constant set-valued functions, then condition (2.1) can be weakened with the following condition

$$
f \leq h+\varepsilon \cdot \mathbf{B}, \quad L(h)\left(x_{0}\right) \subset L(f)\left(x_{0}\right)+\varepsilon \cdot \mathbf{B} .
$$

Obviously, the identity operator $I: \mathcal{C} \rightarrow \mathcal{C}$ satisfies conditions a) and b) of Theorem 2.3. Theorems 2.2 and 2.3 hold also if we replace $\mathcal{K}\left(\mathbb{R}^{d}\right)$ with $\mathcal{K}(E)$, where $E$ is a Fréchet space. However, in the setting of finite dimensional spaces, we can add the following new criteria for Korovkin systems for the identity operator.

Theorem 2.4. Let $(X, \sigma)$ be a compact metric space, $\mathcal{C}$ a subcone of $C\left(X, \mathcal{K}\left(\mathbb{R}^{d}\right)\right)$ and $H$ a subset of $\mathcal{C}$ which contains the functions

$$
x \mapsto K+\lambda \sigma\left(x, x_{0}\right) \cdot \boldsymbol{B}
$$

whenever $K \in \mathcal{K}\left(\mathbb{R}^{d}\right), x_{0} \in X$ and $\lambda \geq 0$ (in particular, $H$ contains the constant functions). Then, $H$ is a Korovkin system in $\mathcal{C}$. 
Proof. Let $f \in \mathcal{C}$ and $x_{0} \in X$. Since $f$ is Hausdorff continuous and $X$ is compact, we can find $M>0$ such that, for every $x \in X, f(x) \subset f\left(x_{0}\right)+M \cdot \mathbf{B}$. Now, let $\varepsilon>0$; from the Hausdorff continuity of $f$, we can find $\delta>0$ such that, for every $x \in X$ satisfying $\sigma\left(x, x_{0}\right) \leq \delta$, we have $f(x) \subset f\left(x_{0}\right)+\frac{\varepsilon}{2} \cdot \mathbf{B}$. Now, consider the function $h: X \rightarrow \mathcal{K}\left(\mathbb{R}^{d}\right)$ defined by setting, for every $x \in X$,

$$
h(x):=f\left(x_{0}\right)+\frac{\varepsilon}{2} \cdot \mathbf{B}+\frac{\sigma\left(x, x_{0}\right)}{\delta} \cdot \mathbf{B} .
$$

The function $h$ is obviously Hausdorff continuous and our assumptions ensure that $h \in H \subset \mathcal{C}$. Now, we show that $f \leq h$. Indeed, if $\sigma\left(x, x_{0}\right) \leq \delta$, we have

$$
f(x) \subset f\left(x_{0}\right)+\frac{\varepsilon}{2} \cdot \mathbf{B} \subset h(x)
$$

and similarly, if $\sigma\left(x, x_{0}\right)>\delta$, we have

$$
f(x) \subset f\left(x_{0}\right)+M \cdot \mathbf{B} \subset f\left(x_{0}\right)+\frac{\varepsilon}{2} \cdot \mathbf{B}+\frac{\sigma\left(x, x_{0}\right)}{\delta} \cdot \mathbf{B}=h(x) .
$$

Finally, we obviously have

$$
h\left(x_{0}\right)=f\left(x_{0}\right)+\frac{\varepsilon}{2} \cdot \mathbf{B} .
$$

Hence, the subset $H$ satisfies the assumptions in Theorem 2.3 and therefore is a Korovkin system in $\mathcal{C}$.

The proof of the following result is similar by considering the function $k: X \rightarrow \mathcal{K}\left(\mathbb{R}^{d}\right)$ defined by setting, for every $x \in X$,

$$
k(x):=f\left(x_{0}\right)+\frac{\varepsilon}{2} \cdot \mathbf{B}+\frac{\sigma\left(x, x_{0}\right)^{2}}{\delta^{2}} \cdot \mathbf{B}
$$

in place of $h$. We omit the details for the sake of brevity.

Theorem 2.5. Let $(X, \sigma)$ be a compact metric space, $\mathcal{C}$ a subcone of $C\left(X, \mathcal{K}\left(\mathbb{R}^{d}\right)\right)$ and $H$ a subset of $\mathcal{C}$ which contains the functions

$$
x \mapsto K+\lambda \sigma\left(x, x_{0}\right)^{2} \cdot \boldsymbol{B}
$$

whenever $K \in \mathcal{K}\left(\mathbb{R}^{d}\right), x_{0} \in X$ and $\lambda \geq 0$. Then, $H$ is a Korovkin system in $\mathcal{C}$.

As a particular case, we can state the preceding Theorems 2.4 and 2.5 in the case where $X$ is a compact subset of $\mathbb{R}$.

Corollary 2.1. Let $X$ be a compact subset of $\mathbb{R}, \mathcal{C}$ a subcone of $C\left(X, \mathcal{K}\left(\mathbb{R}^{d}\right)\right)$ and $H$ a subset of $\mathcal{C}$ which contains the functions

$$
x \mapsto K+\lambda\left|x-x_{0}\right| \cdot \boldsymbol{B}
$$

(or alternatively, contains the functions

$$
\left.x \mapsto K+\lambda\left(x-x_{0}\right)^{2} \cdot \boldsymbol{B}\right)
$$

whenever $K \in \mathcal{K}\left(\mathbb{R}^{d}\right), x_{0} \in X$ and $\lambda \geq 0$ (in particular, $H$ contains the constant functions). Then, $H$ is a Korovkin system in $\mathcal{C}$.

In particular, if $X=[0,1]$ and $d=1$, we obtain that the subcone $H$ of $C([0,1], \mathcal{K}(\mathbb{R}))$ containing the functions

$$
x \mapsto\left(\lambda+\mu\left|x-x_{0}\right|\right) \cdot[-1,1]
$$

for every $x_{0} \in[0,1]$, is a Korovkin system in $C([0,1], \mathcal{K}(\mathbb{R}))$. In the alternative formulation, we have to require that the functions

$$
x \mapsto\left(\lambda+\mu\left(x-x_{0}\right)^{2}\right) \cdot[-1,1], \quad x_{0} \in[0,1],
$$


belong to $H$, and hence we find the classical Korovkin system in $C([0,1], \mathcal{K}(\mathbb{R}))$ consisting of the functions

$$
x \mapsto \mathbf{B}, \quad x \mapsto x \cdot \mathbf{B}, \quad x \mapsto x^{2} \cdot \mathbf{B} .
$$

We conclude this section with the following result which generalizes Theorem 2.3. It has been established in [8, Theorem 1.3] only in the particular case of the identity operator. Here, we give a proof in the general case.

Theorem 2.6. Let $X$ be a compact Hausdorff topological space, $\mathcal{C}$ a subcone of $C\left(X, \mathcal{K}\left(\mathbb{R}^{d}\right)\right)$ containing the set

$$
C(X, \mathbb{R}) \otimes \mathcal{K}\left(\mathbb{R}^{d}\right):=\left\{\varphi \cdot A \mid \varphi \in C\left(X, \mathbb{R}^{d}\right), A \in \mathcal{K}\left(\mathbb{R}^{d}\right)\right\}
$$

and $L: \mathcal{C} \rightarrow \mathcal{C}$ a continuous monotone linear operator. If $H$ is a subset of $\mathcal{C}$ such that, for every $f \in \mathcal{C}$, $x_{0} \in X$ and $\varepsilon>0$, there exist $h_{1}, \ldots, h_{m} \in H$ such that

$$
f \leq h_{j}, j=1, \ldots, m, \quad \bigcap_{j=1}^{m} L\left(h_{j}\right)\left(x_{0}\right) \subset L(f)\left(x_{0}\right)+\varepsilon \cdot \boldsymbol{B},
$$

then $H$ is a Korovkin set for $L$ in $\mathcal{C}$.

Proof. Let $\left(L_{i}\right)_{i \in I}$ be an equicontinuous net of convexity monotone linear operators such that $\lim _{i \in I}^{\leq} L_{i}(h)=L(h)$ for every $h \in H$. Let $f \in \mathcal{C}$.

First step. Assume that $f=\{\varphi\}$ with $\varphi \in C\left(X, \mathbb{R}^{d}\right)$. Let $\varepsilon>0$ and $x_{0} \in X$. The assumptions on $H$ ensure the existence of $h_{1}, \ldots, h_{m} \in H$ such that

$$
f \leq h_{j}, j=1, \ldots, m, \quad \bigcap_{j=1}^{m} L\left(h_{j}\right)\left(x_{0}\right) \subset L(f)\left(x_{0}\right)+\frac{\varepsilon}{4} \cdot \mathbf{B} .
$$

Since $L(f)$ and each $L\left(h_{j}\right), j=1, \ldots, m$ are Hausdorff continuous at $x_{0}$, there exists a neighborhood $U$ of $x_{0}$ such that, for every $x \in U$,

$$
L(f)\left(x_{0}\right) \subset L(f)(x)+\frac{\varepsilon}{4} \cdot \mathbf{B}, \quad L\left(h_{j}\right)(x) \subset L\left(h_{j}\right)\left(x_{0}\right)+\frac{\varepsilon}{4} \cdot \mathbf{B}, j=1, \ldots, m .
$$

Hence, for every $x \in U$, we have

$$
\bigcap_{j=1}^{m} L\left(h_{j}\right)(x) \subset \bigcap_{j=1}^{m} L\left(h_{j}\right)\left(x_{0}\right)+\frac{\varepsilon}{4} \cdot \mathbf{B} \subset L(f)\left(x_{0}\right)+\frac{\varepsilon}{2} \cdot \mathbf{B} \subset L(f)(x)+\frac{3 \varepsilon}{4} \cdot \mathbf{B} .
$$

Since $\lim _{i \in I}^{\leq} L_{i}\left(h_{j}\right)=L\left(h_{j}\right)$ for every $j=1, \ldots, m$, there exists $\alpha \in I$ such that, for every $i \in I$, $i \geq \alpha, j=1 \ldots, m$ and $x \in X$,

$$
L_{i}\left(h_{j}\right)(x) \subset L\left(h_{j}\right)(x)+\frac{\varepsilon}{4} \cdot \mathbf{B}, \quad L\left(h_{j}\right)(x) \subset L_{i}\left(h_{j}\right)(x)+\frac{\varepsilon}{4} \cdot \mathbf{B} .
$$

It follows, for every $i \in I, i \geq \alpha, j=1 \ldots, m$ and $x \in U$,

$$
L_{i}(f)(x) \subset L_{i}\left(h_{j}\right)(x) \subset L\left(h_{j}\right)(x)+\frac{\varepsilon}{4} \cdot \mathbf{B}
$$

and hence

$$
L_{i}(f)(x) \subset \bigcap_{j=1}^{m} L\left(h_{j}\right)(x)+\frac{\varepsilon}{4} \cdot \mathbf{B} \subset L(f)(x)+\varepsilon \cdot \mathbf{B} .
$$

Since $X$ is compact, we can deduce the existence of $\beta \in I$ such that $L_{i}(f)(x) \subset L(f)(x)+\varepsilon \cdot \mathbf{B}$ for every $i \in I, i \geq \beta$. Since $f$ is single-valued, we have also $L(f)(x) \subset L_{i}(f)(x)+\varepsilon \cdot \mathbf{B}$ for every $i \in I, i \geq \beta$ and the proof is complete in this case. 
Second step. Assume that $f=\varphi \cdot A$ with $\varphi \in C(X, \mathbb{R})$ and $A \in \mathcal{K}\left(\mathbb{R}^{d}\right)$. Let $\varepsilon>0$ and $x_{0} \in X$. Since $A$ is compact, there exist $y_{1}, \ldots, y_{p} \in A$ such that

$$
f\left(x_{0}\right)=\varphi\left(x_{0}\right) \cdot A \subset \bigcup_{s=1}^{p} \varphi\left(x_{0}\right) \cdot\left(\left\{y_{s}\right\}+\frac{\varepsilon}{4} \cdot \mathbf{B}\right)
$$

for every $s=1, \ldots, p$, we consider the set-valued function $g_{s}=\left\{\varphi \cdot y_{s}\right\}$ which satisfies $g_{s} \leq f$. From the assumptions on $H$, there exist of $h_{1}, \ldots, h_{m} \in H$ such that

$$
f \leq h_{j}, j=1, \ldots, m, \quad \bigcap_{j=1}^{m} L\left(h_{j}\right)\left(x_{0}\right) \subset L(f)\left(x_{0}\right)+\frac{\varepsilon}{4} \cdot \mathbf{B} .
$$

Since $L(f)$ and each $L\left(g_{s}\right), s=1, \ldots, p$ and $L\left(h_{j}\right), j=1, \ldots, m$ are Hausdorff continuous at $x_{0}$, we can apply the same argument of the first step and obtain a neighborhood $U$ of $x_{0}$ such that, for every $x \in U$,

$$
L(f)(x) \subset \bigcup_{s=1}^{p} L\left(g_{s}\right)(x)+\frac{3 \varepsilon}{4} \cdot \mathbf{B}, \quad \bigcap_{j=1}^{m} L\left(h_{j}\right)(x) \subset L(f)(x)+\frac{3 \varepsilon}{4} \cdot \mathbf{B} .
$$

For every $s=1, \ldots, p$, the function $g_{s}$ is single-valued and therefore the net $\left(L_{i}\left(g_{s}\right)\right)_{i \in I}$ converges to $L\left(g_{s}\right)$. Moreover, $\lim _{i \in I}^{\leq} L_{i}\left(h_{j}\right)=L\left(h_{j}\right)$ for every $j=1, \ldots, m$ and therefore we can find $\alpha \in I$ such that, for every $i \in I, i \geq \alpha, s=1, \ldots, p, j=1, \ldots, m$ and $x \in X$,

$$
L_{i}\left(h_{j}\right)(x) \subset L\left(h_{j}\right)(x)+\frac{\varepsilon}{4} \cdot \mathbf{B}, \quad L\left(g_{s}\right)(x) \subset L_{i}\left(g_{s}\right)(x)+\frac{\varepsilon}{4} \cdot \mathbf{B} \subset L_{i}(f)(x)+\frac{\varepsilon}{4} \cdot \mathbf{B} .
$$

It follows, for every $i \in I, i \geq \alpha, j=1, \ldots, m$ and $x \in U$,

$$
L(f)(x) \subset \bigcup_{s=1}^{p} L\left(g_{s}\right)(x)+\frac{3 \varepsilon}{4} \cdot \mathbf{B} \subset L_{i}(f)(x)+\varepsilon \cdot \mathbf{B} .
$$

Similarly, since $L_{i}(f)(x) \subset L_{i}\left(h_{j}\right)(x) \subset L\left(h_{j}\right)+\frac{3 \varepsilon}{4} \cdot \mathbf{B}$, we have

$$
L_{i}(f)(x) \subset \bigcap_{j=1}^{m} L\left(h_{j}\right)(x)+\frac{\varepsilon}{4} \cdot \mathbf{B} \subset L(f)(x)+\varepsilon \cdot \mathbf{B} .
$$

Since $X$ is compact, we can conclude the proof as in the first step.

Third step. Let $f \in \mathcal{C}$. From [8, Lemma 1.2], for every $\varepsilon>0$, we can find $\varphi_{1}, \ldots, \varphi_{p} \in C(X, \mathbb{R})$ and $A_{1}, \ldots, A_{p} \in \mathcal{K}\left(\mathbb{R}^{d}\right)$ such that

$$
f \leq \sum_{s=1}^{p} \varphi_{s} \cdot A_{s}+\varepsilon \cdot \mathbf{B}, \quad \sum_{s=1}^{p} \varphi_{s} \cdot A_{s}+\varepsilon \cdot \mathbf{B} \leq f,
$$

and from the second step we easily obtain the convergence of $\left(L_{i}(f)\right)_{i \in I}$ to $L(f)$ also in this case.

\section{CONVEXITY MONOTONE OPERATORS AND KOROVKIN APPROXIMATION OF VECTOR-VALUED FUNCTIONS}

Korovkin approximation of set-valued functions is strictly related to Korovkin approximation of single vector-valued functions. In this section, we consider a particular class of setvalued and vector-valued operators which will allow us to state some applications of the results in Section 2 concerning vector-valued functions and conversely to deepen the analysis of set-valued function in the next section. First of all, let $X$ be a Hausdorff compact topological 
space and let $T: C\left(X, \mathbb{R}^{d}\right) \rightarrow C\left(X, \mathbb{R}^{d}\right)$ a linear operator. We recall that $T$ is called a convexity monotone operator if it satisfies the following property, for every $\varphi_{1}, \varphi_{2} \in C\left(X, \mathbb{R}^{d}\right)$ having disjoint graphs

$$
\varphi \in \operatorname{co}\left(\varphi_{1}, \varphi_{2}\right) \Rightarrow T(\varphi) \in \operatorname{co}\left(T\left(\varphi_{1}\right), T\left(\varphi_{2}\right)\right) .
$$

A simple equivalent formulation of the preceding definition states that $T$ is convexity monotone if and only if for every $\varphi, \varphi_{1} \in C\left(X, \mathbb{R}^{d}\right)$ such that $\varphi_{1}(x) \neq 0$ for every $x \in X$, we have (see [9, Proposition 2.4])

$$
\varphi \in \operatorname{co}\left(0, \varphi_{1}\right) \Rightarrow T(\varphi) \in \operatorname{co}\left(0, T\left(\varphi_{1}\right)\right) .
$$

The convexity monotone property generalizes that of positive operator in the case $d=1$. In this regard, different notions have been introduced with the same aim. In [16], Nishishiraho introduced the quasi-positive operators; namely a linear operator $T: C\left(X, \mathbb{R}^{d}\right) \rightarrow C\left(X, \mathbb{R}^{d}\right)$ is said to be quasi positive if, for every $\varphi, \psi \in C(X, \mathbb{R})$ such that $|\varphi| \leq \psi$, we have

$$
\|T(\varphi \cdot y)(x)\| \leq\|T(\psi \cdot y)(x)\|
$$

for every $x \in X$ and $y \in \mathbb{R}^{d}$. The connections between convexity monotone and quasi positive operators have been investigated in $[7,9]$. In general, convexity monotone operators are quasi-positive operators ([9, Proposition 2.6]); in the case $d=1$, we have the following characterization [7, Proposition 2.5].

Proposition 3.1. Let $X$ a connected compact Hausdorff topological space and $T: C(X, \mathbb{R}) \rightarrow C(X, \mathbb{R})$ a continuous linear operator. Then, the following statements are equivalent:

a) $T$ is convexity monotone.

b) $T$ is quasi-positive.

c) There exist two closed subsets $X_{T}^{+}$and $X_{T}^{-}$of $X$ such that $X=X_{T}^{+} \cup X_{T}^{-}$and

$$
\varphi \in C(X, \mathbb{R}), \varphi \geq 0 \Rightarrow T(\varphi) \geq 0 \text { on } X_{T}^{+}, T(\varphi) \leq 0 \text { on } X_{T}^{-} .
$$

d) There exist two closed subsets $X_{T}^{+}$and $X_{T}^{-}$of $X$ such that $X=X_{T}^{+} \cup X_{T}^{-}$and

$$
\varphi, \psi \in C(X, \mathbb{R}), \varphi \leq \psi \Rightarrow T(\varphi) \leq T(\psi) \text { on } X_{T}^{+}, T(\varphi) \geq T(\psi) \text { on } X_{T}^{-} .
$$

If $T: C(X, \mathbb{R}) \rightarrow C(X, \mathbb{R})$ is convexity monotone, the two subsets $X_{T}^{+}$and $X_{T}^{-}$provided in c) and $\mathrm{d}$ ) of the preceding proposition are given by

$$
X_{T}^{+}:=\{x \in X \mid L(\mathbf{1})(x) \geq 0\}, \quad X_{T}^{-}:=\{x \in X \mid L(\mathbf{1})(x) \leq 0\} .
$$

We observe that if $x \in X_{T}^{+} \cap X_{T}^{-}$, we have necessarily $T(\xi)(x)=0$ for every $\xi \in C([0,1], \mathbb{R})$. Condition c) in Proposition 3.1 has been used in [10] in order to extend the results in [12, 15] to sequences of convexity monotone operators (not necessarily positive) in the space $C([0,1])$. Our aim is a more general extension in the case where $X$ is not necessarily the interval $[0,1]$. Classical examples of convexity monotone operators $T: C([a, b]) \rightarrow C([a, b])$ which are not positive are integral operators $T(\xi)(x):=\int_{x_{0}}^{x} \xi$ with $\left.x_{0} \in\right] a, b\left[\right.$ (in this case $X_{T}^{+}=\left[a, x_{0}\right]$ and $X_{T}^{-}=$ $\left.\left[x_{0}, b\right]\right)$. The notion of convexity monotone operator introduced in $[5,6]$ for operators in spaces of vector-valued functions can be also considered for operators in the setting of set-valued functions. Namely, a continuous monotone linear operator $L: C\left(X, \mathcal{K}\left(\mathbb{R}^{d}\right)\right) \rightarrow C\left(X, \mathcal{K}\left(\mathbb{R}^{d}\right)\right)$ is said to be convexity monotone if it satisfies the condition

$$
\varphi \in \operatorname{co}\left(\varphi_{1}, \varphi_{2}\right) \Rightarrow L(\{\varphi\}) \subset \operatorname{co}\left(L\left(\left\{\varphi_{1}\right\}\right), L\left(\left\{\varphi_{2}\right\}\right)\right)
$$

for every $\varphi_{1}, \varphi_{2} \in C\left(X, \mathbb{R}^{d}\right)$ having disjoint graphs. The preceding condition is equivalent to the following, for every $\varphi \in C\left(X, \mathbb{R}^{d}\right)$,

$$
L(\operatorname{co}(0, \varphi)) \subset \operatorname{co}(0, L(\{\varphi\})) .
$$


We begin by stating some results obtained in $[6,7,8]$. We recall that $\varphi_{1}, \ldots, \varphi_{n}$ are affinely independent if, for every $x \in X$, we have

$$
\lambda_{1}, \ldots, \lambda_{n} \in[0,1], \sum_{i=1}^{n} \lambda_{i}=0, \sum_{i=1}^{n} \lambda_{i} \varphi_{i}(x)=0 \Rightarrow \lambda_{1}=0, \ldots, \lambda_{n}=0 .
$$

If $\mathcal{C}$ is a subcone of $C\left(X, \mathcal{K}\left(\mathbb{R}^{d}\right)\right)$ and $L: \mathcal{C} \rightarrow \mathcal{C}$ is a convexity monotone linear continuous operator, a subset $H$ of $\mathcal{C}$ is called a Korovkin system in $\mathcal{C}$ with respect to convexity monotone operators for $L$ if for every equicontinuous net $\left(L_{i}\right)_{i \in I}^{\leq}$of convexity monotone linear operators from $\mathcal{C}$ into itself satifying $\lim _{i \in I}^{\leq} L_{i}(h)=L(h)$ for every $h \in H$, we also have $\lim _{i \in I}^{\leq} L_{i}(f)=L(f)$ for every $f \in \mathcal{C}$. Similarly, a subset $M$ of $C\left(X, \mathbb{R}^{d}\right)$ is called a Korovkin system in $C\left(X, \mathbb{R}^{d}\right)$ with respect to convexity monotone operators for a convexity monotone linear continuous operator $T: C\left(X, \mathbb{R}^{d}\right) \rightarrow C\left(X, \mathbb{R}^{d}\right)$ if for every equicontinuous net $\left(T_{i}\right)_{i \in I}^{\leq}$of convexity monotone linear operators from $C\left(X, \mathbb{R}^{d}\right)$ into itself satifying $\lim _{i \in I}^{\leq} T_{i}(\varphi)=T(\varphi)$ for every $\varphi \in M$, we also have $\lim _{i \in I}^{\leq} T_{i}(\varphi)=T(\varphi)$ for every $\varphi \in C\left(X, \mathbb{R}^{d}\right)$.

Theorem 3.7. ([7, Theorem 2.6]) Let $X$ be a connected compact Hausdorff topological space, $T$ : $C\left(X, \mathbb{R}^{d}\right) \rightarrow C\left(X, \mathbb{R}^{d}\right)$ a convexity monotone linear operator and $M$ a subset of $C\left(X, \mathbb{R}^{d}\right)$ such that, for every $\varphi \in C\left(X, \mathbb{R}^{d}\right), x_{0} \in X$ and $\varepsilon>0$, there exist affinely independent functions $\varphi_{1}, \ldots, \varphi_{m} \in$ $C\left(X, \mathbb{R}^{d}\right)$ satisfying the following conditions

$$
\begin{gathered}
\varphi \in \operatorname{co}\left(\varphi_{1}, \ldots, \varphi_{m}\right), \\
\operatorname{co}\left(T\left(\varphi_{1}\right)\left(x_{0}\right), \ldots, T\left(\varphi_{m}\right)\left(x_{0}\right)\right) \subset T(\varphi)\left(x_{0}\right)+\varepsilon \cdot \boldsymbol{B} .
\end{gathered}
$$

Then, $M$ is a Korovkin system in $C\left(X, \mathbb{R}^{d}\right)$ for $T$ with respect to convexity monotone operators.

We state some consequences of the preceding result. In the case $d=1$, the following result generalizes a classical property of Korovkin systems obtained by Berens and Lorentz [3] in the case of the identity operator and by Ferguson and Rusk [11] in the case of positive operators (see also [1, Chapter 3]).

Corollary 3.2. ([7, Corollaries 2.8]) Let $X$ be a connected compact Hausdorff topological space, $T$ : $C(X, \mathbb{R}) \rightarrow C(X, \mathbb{R})$ a convexity monotone linear operator and $M$ a subset of $C(X, \mathbb{R})$ such that, for every $\varphi \in C(X, \mathbb{R}), x_{0} \in X$ and $\varepsilon>0$, there exist $\psi, \chi \in C(X, \mathbb{R})$ satisfying the following conditions

$$
\psi \leq \varphi \leq \chi, \quad\left|T(\chi)\left(x_{0}\right)-T(\psi)\left(x_{0}\right)\right| \leq \varepsilon .
$$

Then, $M$ is a Korovkin system in $C(X, \mathbb{R})$ for $T$ with respect to convexity monotone operators.

In the particular case where $T$ is the identity operator, we have the following further consequences.

Corollary 3.3. ([7, Corollaries 2.7, 2.9] and [8, Corollary 3.3]) Let $X$ be a connected compact Hausdorff topological space and $\Gamma$ a Korovkin set in $C(X, \mathbb{R})$. Then, the following subsets of $C\left(X, \mathbb{R}^{d}\right)$ :

$$
\begin{gathered}
M_{1}:=\left\{\varphi \cdot y \mid \varphi \in \Gamma, y \in \mathbb{R}^{d}\right\}, \\
M_{2}:=\left\{\left(\delta_{i, 1} \varphi, \ldots, \delta_{i, d} \varphi\right) \mid i=1, \ldots, d, \varphi \in \Gamma\right\}
\end{gathered}
$$

$\left(\delta_{i, j}\right.$ denotes as usual the Kronecker symbol) are Korovkin systems in $C\left(X, \mathbb{R}^{d}\right)$ for the identity operator with respect to convexity monotone operators.

The next result is a set-valued version of Theorem 3.7. 
Theorem 3.8. Let $X$ be a connected compact Hausdorff topological space, $\mathcal{C}$ a subcone of $C\left(X, \mathcal{K}\left(\mathbb{R}^{d}\right)\right)$ containing the single-valued continuous functions and the constant function $1 \cdot \boldsymbol{B}$ and $L: \mathcal{C} \rightarrow \mathcal{C} a$ convexity monotone continuous linear operator satisfying conditions $a$ ) and $b$ ) of Theorem 2.3. Let $H$ be a subset of $\mathcal{C}$ containing the function $\boldsymbol{B}$ and such that, for every $f \in \mathcal{C}, x_{0} \in X$ and $\varepsilon>0$, there exist affinely independent functions $\varphi_{1}, \ldots, \varphi_{m} \in C\left(X, \mathbb{R}^{d}\right)$ satisfying the following conditions

$$
\left.f \leq \operatorname{co}\left(\varphi_{1}, \ldots, \varphi_{m}\right) \quad \text { (i.e., } \forall x \in X: f(x) \subset \operatorname{co}\left(\varphi_{1}(x), \ldots, \varphi_{m}(x)\right)\right)
$$

and

$$
\operatorname{co}\left(L\left(\left\{\varphi_{1}\right\}\right)\left(x_{0}\right), \ldots, L\left(\left\{\varphi_{m}\right\}\right)\left(x_{0}\right)\right) \subset L(\{\varphi\})\left(x_{0}\right)+\varepsilon \cdot \boldsymbol{B} .
$$

Then, $H$ is a Korovkin system in $\mathcal{C}$ for $L$ with respect to convexity monotone operators.

Proof. Let $\left(L_{i}\right)_{i \in I}$ be an equicontinuous net of convexity monotone linear operators converging to $L(h)$ for every $h \in H$. First, we show that if $\varphi_{1}, \ldots, \varphi_{m}$ are affinely independent functions in $C\left(X, \mathbb{R}^{d}\right)$, then

$$
\lim _{i \in I} \leq L_{i}\left(\operatorname{co}\left(\varphi_{1}, \ldots, \varphi_{m}\right)\right)=L\left(\operatorname{co}\left(\varphi_{1}, \ldots, \varphi_{m}\right)\right) .
$$

Let $\varepsilon>0$; since $\left(L_{i}(\mathbf{B})\right)_{i \in I}$ converges to $L(\mathbf{B})$, we can find $M \geq 1$ such that

$$
L_{i}(1 \cdot \mathbf{B}) \subset M \cdot \mathbf{B}, \quad L(1 \cdot \mathbf{B}) \subset M \cdot \mathbf{B} .
$$

For every $x \in X$, the set $\operatorname{co}\left(\varphi_{1}(x), \ldots, \varphi_{m}(x)\right)$ is compact and therefore there exist $\lambda(s):=$ $\left(\lambda_{1}(s), \ldots, \lambda_{m}(s)\right), s=1, \ldots, p$, such that $\lambda_{j}(s) \geq 0$ for every $j=1, \ldots, m$ and $\sum_{j=1}^{p} \lambda_{j}(s)=1$ for every $s=1, \ldots, p$ and further

$$
\operatorname{co}\left(\varphi_{1}(x), \ldots, \varphi_{m}(x)\right) \subset \bigcup_{s=1}^{p}\left(\sum_{j=1}^{m} \lambda_{j}(s)\left\{\varphi_{j}(x)\right\}+\frac{\varepsilon}{2 M} \cdot \mathbf{B}\right) .
$$

Since $X$ is compact, the preceding formula can be extended to every $x \in X$. From Theorem 3.7, we have the convergence of $\left(L_{i}(\{\varphi\})\right)_{i \in I}$ to $L(\{\varphi\})$ for every $\varphi \in C\left(X, \mathbb{R}^{d}\right)$ and consequently the nets $\left(\sum_{j=1}^{m} \lambda_{j}(s) L_{i}\left(\left\{\varphi_{j}\right\}\right)\right)_{i \in I}$ converge to $\sum_{j=1}^{m} \lambda_{j}(s) L\left(\left\{\varphi_{j}\right\}\right)$ for every $s=$ $1, \ldots, p$. Hence, we can find $\alpha \in I$ such that, for every $i \in I, i \geq \alpha$ and $s=1, \ldots, p$,

$$
\begin{aligned}
& \sum_{j=1}^{m} \lambda_{j}(s) L\left(\left\{\varphi_{j}\right\}\right) \subset \sum_{j=1}^{m} \lambda_{j}(s) L_{i}\left(\left\{\varphi_{j}\right\}\right)+\frac{\varepsilon}{2} \cdot \mathbf{B}, \\
& \sum_{j=1}^{m} \lambda_{j}(s) L_{i}\left(\left\{\varphi_{j}\right\}\right) \subset \sum_{j=1}^{m} \lambda_{j}(s) L\left(\left\{\varphi_{j}\right\}\right)+\frac{\varepsilon}{2} \cdot \mathbf{B} .
\end{aligned}
$$

It follows, for every $i \in I, i \geq \alpha$,

$$
\begin{aligned}
L\left(\operatorname{co}\left(\varphi_{1}, \ldots, \varphi_{m}\right)\right) & \subset \bigcup_{s=1}^{p}\left(\sum_{j=1}^{m} \lambda_{j}(s) L\left(\left\{\varphi_{j}\right\}\right)+L\left(\frac{\varepsilon}{2 M} \cdot \mathbf{B}\right)\right) \\
& \subset \bigcup_{s=1}^{p}\left(\sum_{j=1}^{m} \lambda_{j}(s) L\left(\left\{\varphi_{j}\right\}\right)+\frac{\varepsilon}{2} \cdot \mathbf{B}\right) \\
& \subset \bigcup_{s=1}^{p}\left(\sum_{j=1}^{m} \lambda_{j}(s) L_{i}\left(\left\{\varphi_{j}\right\}\right)+\varepsilon \cdot \mathbf{B}\right) \\
& \subset L_{i}\left(\operatorname{co}\left(\varphi_{1}, \ldots, \varphi_{m}\right)\right)+\varepsilon \cdot \mathbf{B},
\end{aligned}
$$


and conversely

$$
\begin{aligned}
L_{i}\left(\operatorname{co}\left(\varphi_{1}, \ldots, \varphi_{m}\right)\right) & \subset \bigcup_{s=1}^{p}\left(\sum_{j=1}^{m} \lambda_{j}(s) L_{i}\left(\left\{\varphi_{j}\right\}\right)+L_{i}\left(\frac{\varepsilon}{2 M} \cdot \mathbf{B}\right)\right) \\
& \subset \bigcup_{s=1}^{p}\left(\sum_{j=1}^{m} \lambda_{j}(s) L_{i}\left(\left\{\varphi_{j}\right\}\right)+\frac{\varepsilon}{2} \cdot \mathbf{B}\right) \\
& \subset \bigcup_{s=1}^{p}\left(\sum_{j=1}^{m} \lambda_{j}(s) L\left(\left\{\varphi_{j}\right\}\right)+\varepsilon \cdot \mathbf{B}\right) \\
& \subset L\left(\operatorname{co}\left(\varphi_{1}, \ldots, \varphi_{m}\right)\right)+\varepsilon \cdot \mathbf{B} .
\end{aligned}
$$

Hence, the net $\left(L_{i}\left(\operatorname{co}\left(\varphi_{1}, \ldots, \varphi_{m}\right)\right)\right)_{i \in I}$ converges to $L\left(\operatorname{co}\left(\varphi_{1}, \ldots, \varphi_{m}\right)\right)$. At this point, we observe that the set

$$
\left\{\operatorname{co}\left(\varphi_{1}, \ldots, \varphi_{m}\right) \mid \varphi_{1}, \ldots, \varphi_{m} \text { affinely independent in } C\left(X, \mathbb{R}^{n}\right)\right\}
$$

satisfies condition (2.1) in Theorem 2.3; hence we can proceed just as in the proof of Theorem 2.3 in order to show the convergence of $\left(L_{i}(f)\right)_{i \in I}$ to $L(f)$ for every $f \in \mathcal{C}$ and this completes the proof.

\section{FURTHER DEVELOPMENTS ON THE APPROXIMATION OF SET-VALUED FUNCTIONS}

In this section, we consider some recent developments of Korovkin approximation theory in the case where the existence of the limit operator is not assigned. The following results have been established in [10] in the case $X=[0,1]$. Here, we consider the more general setting of metric spaces. Let $(X, \sigma)$ be a compact metric space. Functions in $C\left(X, \mathcal{K}\left(\mathbb{R}^{d}\right)\right)$ can be approximated by functions in $C\left(X, \mathbb{R}^{d}\right)$, as shown in the following lemma.

Lemma 4.1. Let $f \in C\left(X, \mathcal{K}\left(\mathbb{R}^{d}\right)\right)$. Then, for every $\varepsilon>0$, there exist $\varphi_{1}, \ldots, \varphi_{m} \in \operatorname{Sel}(f)$ such that, for every $x \in X$,

$$
f(x) \subset \bigcup_{i=1}^{m}\left(\left\{\varphi_{i}(x)\right\}+\varepsilon \cdot \mathbf{B}\right) .
$$

Proof. The proof follows the same line of that of [10, Lemma 2.1], but for the sake of completeness we give some details. Let $\varepsilon>0$ and $x_{0} \in X$. From the compactness of $f\left(x_{0}\right)$, we can find $y_{1}, \ldots, y_{m} \in f\left(x_{0}\right)$ such that

$$
f\left(x_{0}\right) \subset \bigcup_{i=1}^{m}\left(\left\{y_{i}\right\}+\frac{\varepsilon}{3} \cdot \mathbf{B}\right) .
$$

Moreover, from [5, Proposition 1.1], we can find $\varphi_{1}, \ldots, \varphi_{m} \in \operatorname{Sel}(f)$ such that $\varphi_{i}\left(x_{0}\right)=y_{i}$ for every $i=1, \ldots, m$. Since $f$ and $\varphi_{1}, \ldots, \varphi_{m}$ are continuous, there exists $\delta>0$ such that

$$
f(x) \subset f\left(x_{0}\right)+\frac{\varepsilon}{3} \cdot \mathbf{B}, \quad\left\|\varphi_{i}(x)-\varphi_{i}\left(x_{0}\right)\right\| \leq \frac{\varepsilon}{3},
$$

for every $x \in X$ such that $\sigma\left(x, x_{0}\right)<\delta$ and $i=1, \ldots, m$. Hence, for every $x \in X$ such that $\sigma\left(x, x_{0}\right)<\delta$,

$$
f(x) \subset f\left(x_{0}\right)+\frac{\varepsilon}{3} \cdot \mathbf{B} \subset \bigcup_{i=1}^{m}\left(\left\{\varphi_{i}\left(x_{0}\right)\right\}+\frac{2 \varepsilon}{3} \cdot \mathbf{B}\right) \subset \bigcup_{i=1}^{m}\left(\left\{\varphi_{i}(x)\right\}+\varepsilon \cdot \mathbf{B}\right) .
$$

At this point, from a compactness argument on $X$, we obtain the proof. 
We observe that if $\varphi_{1}, \ldots, \varphi_{m} \in C\left(X, \mathbb{R}^{d}\right)$, then the set-valued function $\operatorname{co}\left(\varphi_{1}, \ldots, \varphi_{m}\right) \in$ $C\left(X, \mathcal{K}\left(\mathbb{R}^{d}\right)\right)$ and since $f$ takes convex values, (4.5) can be written as

$$
\operatorname{co}\left(\varphi_{1}, \ldots, \varphi_{m}\right) \leq f \leq \operatorname{co}\left(\varphi_{1}, \ldots, \varphi_{m}\right)+\varepsilon \cdot \mathbf{B} .
$$

In the following proposition, we state some general properties of continuous monotone linear operators on $C\left(X, \mathcal{K}\left(\mathbb{R}^{d}\right)\right)$. We only sketch the proof since it is similar to that of [10, Proposition 2.2].

Proposition 4.2. Let $L: C\left(X, \mathcal{K}\left(\mathbb{R}^{d}\right)\right) \rightarrow C\left(X, \mathcal{K}\left(\mathbb{R}^{d}\right)\right)$ be a continuous monotone linear operator on $C\left(X, \mathcal{K}\left(\mathbb{R}^{d}\right)\right)$. Then,

(i) $L(\{0\})=\{0\}$ and consequently $L$ maps single-valued continuous functions into single-valued continuous functions, i.e., for every $\varphi \in C\left(X, \mathbb{R}^{d}\right)$ there exists $\psi \in C\left(X, \mathbb{R}^{d}\right)$ such that $L(\{\varphi\})=\{\psi\}$

(ii) If $f \in C\left(X, \mathcal{K}\left(\mathbb{R}^{d}\right)\right)$, then

$$
L(f)=\overline{\bigcup_{\varphi \in \operatorname{Sel}(f)} L(\{\varphi\})}=\overline{\bigcup_{\varphi_{1}, \ldots, \varphi_{m} \in \operatorname{Sel}(f)} L\left(\operatorname{co}\left(\varphi_{1}, \ldots, \varphi_{m}\right)\right)},
$$

i.e.,

$$
L(f)(x)=\overline{\bigcup_{\varphi \in \operatorname{Sel}(f)} L(\{\varphi\})(x)}=\overline{\bigcup_{\varphi_{1}, \ldots, \varphi_{m} \in \operatorname{Sel}(f)} L\left(\operatorname{co}\left(\varphi_{1}, \ldots, \varphi_{m}\right)\right)(x)}
$$

for every $x \in X$.

Proof. Obviously $\{0\} \subset L(\{0\})$ and moreover $L(\{0\})=L(\{0\})+L(\{0\})$; hence $L(\{0\})=\{0\}$. Now, let $\varphi \in C\left(X, \mathbb{R}^{d}\right), x \in X$ and $y, z \in\{\varphi\}(x)$. From [4, Proposition 1.1], we can find $\psi_{1}, \psi_{2} \in \operatorname{Sel}(L(\{\varphi\}))$ such that $\psi_{1}(x)=y$ and $\psi_{2}(x)=z$. The linearity of $L$ yields $z-y=$ $\psi_{2}(x)-\psi_{1}(x) \in L(\{\varphi\})+L(\{-\varphi\})=L(\{0\})=\{0\}$ and therefore $y=z$. Thus, $L(\{\varphi\})$ must be single-valued. As regards to the proof of the first equality in (4.7), the monotonicity property of $L$ yields the inequality $\bigcup_{\varphi \in \operatorname{Sel}(f)} L(\{\varphi\}) \leq L(f)$ and since $L(f)(x)$ is closed for every $x \in[0,1]$, we get $\overline{\bigcup_{\varphi \in \operatorname{Sel}(f)} L(\{\varphi\})(x)} \leq L(f)(x)$. In order to show the converse inequality, first observe that since $L$ is continuous and takes compact values on the compact space $X$, there exists $\alpha>0$ such that $L\left(e_{0} \cdot \mathbf{B}\right) \leq \alpha \cdot \mathbf{B}$. If $\varepsilon>0$, from Lemma 4.1, there exist $\varphi_{1}, \ldots, \varphi_{m} \in \operatorname{Sel}(f)$ such that, for every $x \in[0,1]$,

$$
f(x) \subset \bigcup_{i=1}^{m}\left(\left\{\varphi_{i}(x)\right\}+\varepsilon \cdot \mathbf{B}\right)=\left(\bigcup_{i=1}^{m}\left\{\varphi_{i}(x)\right\}\right)+\varepsilon \cdot \mathbf{B} \subset \operatorname{co}\left(\varphi_{1}, \ldots, \varphi_{m}\right)(x)+\varepsilon \cdot \mathbf{B} .
$$

From the monotonicity property of $f$, we obtain

$$
L\left(\operatorname{co}\left(\varphi_{1}, \ldots, \varphi_{m}\right)\right) \leq L(f) \leq L\left(\operatorname{co}\left(\varphi_{1}, \ldots, \varphi_{m}\right)\right)+\varepsilon \alpha \cdot \mathbf{B} .
$$

Since $\operatorname{co}\left(\varphi_{1}, \ldots, \varphi_{m}\right) \subset \bigcup_{\varphi \in \operatorname{Sel}(f)} L(\{\varphi\})$, we have that the uniform distance between $L(f)$ and $\overline{\bigcup_{\varphi \in \operatorname{Sel}(f)} L(\{\varphi\})}$ is less or equal to $\alpha \varepsilon$. From the arbitrarily of $\varepsilon>0$, this distance must be 0 . Hence, the first equality in (4.7) is valid. Finally, the second equality is a consequence of the following inclusions

$$
\bigcup_{\varphi \in \operatorname{Sel}(f)} L(\{\varphi\})(x) \subset \overline{\bigcup_{\varphi_{1}, \ldots, \varphi_{m} \in \operatorname{Sel}(f)} L\left(\operatorname{co}\left(\varphi_{1}, \ldots, \varphi_{m}\right)\right)(x)} \subset L(f)(x)
$$

which are obviously true for every $x \in X$. 
The preceding Proposition 4.2 allows us to associate a single-valued bounded linear operator to every set-valued continuous monotone linear operator. This result has been established in $[10$, Lemma 2.3] in the particular case $X=[0,1]$, while here we consider the general case.

Lemma 4.2. Let $L: C\left(X, \mathcal{K}\left(\mathbb{R}^{d}\right)\right) \rightarrow C\left(X, \mathcal{K}\left(\mathbb{R}^{d}\right)\right)$ be a continuous monotone linear operator. Then, there exists a bounded linear operator $T_{L}: C\left(X, \mathbb{R}^{d}\right) \rightarrow C\left(X, \mathbb{R}^{d}\right)$ such that, for every $\varphi \in C\left(X, \mathbb{R}^{d}\right)$ and $x \in X$,

$$
\left\{T_{L}(\varphi)(x)\right\}=L(\{\varphi\})(x) .
$$

Moreover, if $L: C\left(X, \mathcal{K}\left(\mathbb{R}^{d}\right)\right)$ is convexity monotone, then the operator $T_{L}$ is itself convexity monotone.

The proof is at all similar to that of [10, Lemma 2.3] and therefore we omit the details. We only point out that the operator $T_{L}: C\left(X, \mathbb{R}^{d}\right) \rightarrow C\left(X, \mathbb{R}^{d}\right)$ is defined by setting, for every $\varphi \in C\left(X, \mathbb{R}^{d}\right)$ and $x \in X, T_{L}(\varphi)(x)$ as the unique element of $L(\{\varphi\})(x)$. Conversely, if $T$ : $C\left(X, \mathbb{R}^{d}\right) \rightarrow C\left(X, \mathbb{R}^{d}\right)$ is a bounded linear operator satisfying (3.3), we can define the operator $L_{T}: C\left(X, \mathcal{K}\left(\mathbb{R}^{d}\right)\right) \rightarrow C\left(X, \mathcal{K}\left(\mathbb{R}^{d}\right)\right)$ by setting, for every $f \in C\left(X, \mathcal{K}\left(\mathbb{R}^{d}\right)\right)$ and $x \in X$,

$$
L_{T}(f)(x)=\bigcup_{\varphi \in \operatorname{Sel}(f)}\{T(\varphi)(x)\} .
$$

In [5, Proposition 1.5], it has been shown that $L_{T}(f)(x)$ is a convex compact subset of $\mathbb{R}^{d}$ and $L_{T}(f)$ is continuous and hence $L_{T}$ is well-defined; moreover it has also been shown that $L_{T}$ is a continuous monotone linear operator and satisfies the condition of convexity monotonicity. Now, we are in a position to state the main results of this section which generalizes the Korovkin-type approximation of vector-valued and set-valued functions to the case where the limit operator is not assigned. Some results in this setting have been obtained in [12, 15] and more recently in [10, Theorems 1.1, 1.2 and 2.4]. All the preceding results have been established in the case $X=[0,1]$, while we consider here a non trivial extension to the multivariable case. For the sake of simplicity, we consider the case where $X$ is a compact convex subset of $\mathbb{R}^{2}$. We begin with the case of vector-valued continuous functions by considering first the case of real valued functions and then the general case.

Theorem 4.9. Let $X$ be a convex compact subset of $\mathbb{R}^{2}$ and let $\left(T_{n}\right)_{n \in \mathbb{N}}$ be a sequence of continuous linear operators on $C(X, \mathbb{R})$ satisfying condition (3.3) and assume that

(i) The sequence $\left(T_{n}\left(\mathrm{pr}_{i}^{2}\right)\right)_{n \in \mathbb{N}}, i=1,2$, converges to a real continuous function $h_{i} \in C(X, \mathbb{R})$ ( $\mathrm{pr}_{i}$ denotes the canonical projection $\left(x_{1}, x_{2}\right) \mapsto x_{i}$ of $\mathbb{R}^{2}$ onto $\left.\mathbb{R}\right)$.

(ii) The sequence $\left(\left|T_{n}(\xi)(x, y)\right|\right)_{n \in \mathbb{N}}$ is nonincreasing with respect to $n$ for any convex function $\xi \in C(X, \mathbb{R})$ and $(x, y) \in X$.

Then, there exists a continuous linear operator $T: C(X, \mathbb{R}) \rightarrow C(X, \mathbb{R})$ satisfying condition (3.3) and such that $\lim _{n \rightarrow+\infty} T_{n}(\xi)(x, y)=T(\xi)(x, y)$ for every $\xi \in C(X, \mathbb{R})$ and uniformly with respect to $(x, y) \in X$.

Proof. We observe that the sequence $\left(T_{n}(\xi)\right)_{n \in \mathbb{N}}$ is constant in $n$ for every linear function $\xi$ and therefore converges to a function $T(\xi)=T_{0}(\xi)$; in particular $\left(T_{n}(\mathbf{1})\right)_{n \in \mathbb{N}}$ converges to $T(\mathbf{1})$ and $\left(T_{n}\left(\operatorname{pr}_{i}\right)\right)_{n \in \mathbb{N}}$ converges to $T\left(\operatorname{pr}_{i}\right), i=1,2$. Now, let $X^{+}:=\{(x, y) \in X \mid T(\mathbf{1})(x, y) \geq 0\}$ and $X^{-}:=\{(x, y) \in X \mid T(\mathbf{1})(x, y) \leq 0\}$ and, for every $n \in \mathbb{N}, X_{n}^{+}:=\left\{(x, y) \in X \mid T_{n}(\mathbf{1})(x, y) \geq 0\right\}$ and $X_{n}^{-}:=\left\{(x, y) \in X \mid T_{n}(\mathbf{1})(x, y) \leq 0\right\}$. First, we observe that the sequence $\left(T_{n}\right)_{n \in \mathbb{N}}$ is equibounded. Indeed, if $\xi \in C(X, \mathbb{R})$ is positive and $\|\xi\| \leq 1$, we have $\xi \in \operatorname{co}(0, \mathbf{1})$ and from (3.3) it follows $T_{n}(\xi) \in \operatorname{co}\left(0, T_{n}(\mathbf{1})\right)$ for every $n \in \mathbb{N}$. This yields $0 \leq T_{n}(\xi)(x, y) \leq T_{n}(\mathbf{1})(x, y)$ for every $(x, y) \in X_{n}^{+}$and $T_{n}(\mathbf{1})(x, y) \leq T_{n}(\xi)(x, y) \leq 0$ for every $(x, y) \in X_{n}^{-}$and therefore 
$\left\|T_{n}(\xi)\right\| \leq\left\|T_{n}(\mathbf{1})\right\|$. If $\xi$ is not positive, we apply the above argument to $\xi_{+}$and $\xi_{-}$and we easily obtain $\left\|T_{n}(\xi)\right\|=\left\|T_{n}\left(\xi_{+}\right)-T_{n}\left(\xi_{-}\right)\right\| \leq\left\|T_{n}\left(\xi_{+}\right)\right\|+\left\|T_{n}\left(\xi_{-}\right)\right\| \leq 2\left\|T_{n}(\mathbf{1})\right\|$. Thus

$$
\sup _{n \in \mathbb{N}}\left\|T_{n}\right\| \leq 2 \sup _{n \in \mathbb{N}}\left\|T_{n}\left(T_{\infty}(\mathbf{1})\right)\right\|<+\infty .
$$

Hence, the sequence $\left(T_{n}\right)_{n \in \mathbb{N}}$ is equibounded and therefore it is enough to show the convergence of the sequence $\left(T_{n}(\xi)\right)_{n \in \mathbb{N}}$ for every $\xi \in C^{2}(X, \mathbb{R})$. Let $\xi \in C^{2}(X, \mathbb{R})$ and consider the two functions

$$
\gamma_{ \pm}(x, y):=a\left(x^{2}+y^{2}\right) \pm \xi(x, y), \quad(x, y) \in X
$$

where

$$
a:=\max \left\{\left\|\frac{\partial^{2} \xi}{\partial x^{2}}\right\|,\left\|\frac{\partial^{2} \xi}{\partial y^{2}}\right\|, \sqrt{\frac{1}{2}\left(\left\|\left(\frac{\partial^{2} \xi}{\partial x^{2}}\right)^{2}+\left(\frac{\partial^{2} \xi}{\partial x^{2}}\right)^{2}+\left(\frac{\partial^{2} \xi}{\partial x \partial y}\right)^{2}-\frac{\partial^{2} \xi}{\partial x^{2}} \frac{\partial^{2} \xi}{\partial y^{2}}\right\|\right)}\right\} .
$$

We have, for every $(x, y) \in X$,

$$
\begin{aligned}
& \frac{\partial^{2} \gamma_{ \pm}}{\partial x^{2}}(x, y)=2 a \pm \frac{\partial^{2} \xi}{\partial x^{2}}(x, y) \\
& \frac{\partial^{2} \gamma_{ \pm}}{\partial y^{2}}(x, y)=2 a \pm \frac{\partial^{2} \xi}{\partial y^{2}}(x, y) \\
& \frac{\partial^{2} \gamma_{ \pm}}{\partial x \partial y}(x, y)= \pm \frac{\partial^{2} \xi}{\partial x \partial y}(x, y)
\end{aligned}
$$

and therefore $\frac{\partial^{2} \gamma_{ \pm}}{\partial x^{2}} \geq 0$ and

$$
\begin{aligned}
\frac{\partial^{2} \gamma_{ \pm}}{\partial x^{2}} \frac{\partial^{2} \gamma_{ \pm}}{\partial y^{2}}-\left(\frac{\partial^{2} \gamma_{ \pm}}{\partial x \partial y}\right)^{2} & =\left(2 a \pm \frac{\partial^{2} \xi}{\partial x^{2}}\right)\left(2 a \pm \frac{\partial^{2} \xi}{\partial y^{2}}\right)-\left(\frac{\partial^{2} \xi}{\partial x \partial y}\right)^{2} \\
& =4 a^{2} \pm 2 a\left(\frac{\partial^{2} \xi}{\partial x^{2}}+\frac{\partial^{2} \xi}{\partial y^{2}}\right)+\frac{\partial^{2} \xi}{\partial x^{2}} \frac{\partial^{2} \xi}{\partial y^{2}}-\left(\frac{\partial^{2} \xi}{\partial x \partial y}\right)^{2} \\
& =a^{2} \pm 2 a \frac{\partial^{2} \xi}{\partial x^{2}}+\left(\frac{\partial^{2} \xi}{\partial x^{2}}\right)^{2} \\
& +a^{2} \pm 2 a \frac{\partial^{2} \xi}{\partial y^{2}}+\left(\frac{\partial^{2} \xi}{\partial y^{2}}\right)^{2} \\
& +2 a^{2}+\frac{\partial^{2} \xi}{\partial x^{2}} \frac{\partial^{2} \xi}{\partial y^{2}}-\left(\frac{\partial^{2} \xi}{\partial x \partial y}\right)^{2}-\left(\frac{\partial^{2} \xi}{\partial x^{2}}\right)^{2}-\left(\frac{\partial^{2} \xi}{\partial y^{2}}\right)^{2} \\
& =\left(a \pm \frac{\partial^{2} \xi}{\partial x^{2}}\right)^{2}+\left(a \pm \frac{\partial^{2} \xi}{\partial y^{2}}\right)^{2} \\
& +2 a^{2}+\frac{\partial^{2} \xi}{\partial x^{2}} \frac{\partial^{2} \xi}{\partial y^{2}}-\left(\frac{\partial^{2} \xi}{\partial x \partial y}\right)^{2}-\left(\frac{\partial^{2} \xi}{\partial x^{2}}\right)^{2}-\left(\frac{\partial^{2} \xi}{\partial y^{2}}\right)^{2} \\
& \geq 0 .
\end{aligned}
$$

Hence, the functions $\gamma_{ \pm}$are convex and from assumption (ii), it follows that the sequences $\left(\left|T_{n}\left(\gamma_{ \pm}\right)(x, y)\right|\right)_{n \in \mathbb{N}}$ are nonincreasing in $n$ for every $(x, y) \in X$. Let $(x, y) \in X ;$ if $T(\mathbf{1})(x, y)>$ 0 , we have $(x, y) \in X^{+}$and moreover, taking into account that the sequence $\left(T_{n}(\mathbf{1})\right)_{n \in \mathbb{N}}$ is constant, we also have $(x, y) \in X_{n}^{+}$for every $n \in \mathbb{N}$. Hence, for every $n, p \in \mathbb{N}$, we have 


$$
\begin{aligned}
T_{n}\left(\gamma_{ \pm}\right)(x, y)-T_{n+p}\left(\gamma_{ \pm}\right)(x, y) & \geq 0, i=1,2 \text { and consequently } \\
\left|\left(T_{n}-T_{n+p}\right)(\xi)(x, y)\right| & \leq\|\xi\|\left(T_{n}-T_{n+p}\right)(\mathbf{1})(x, y)+a\left(T_{n}-T_{n+p}\right)\left(\mathrm{pr}_{1}^{2}\right)(x, y) \\
& +a\left(T_{n}-T_{n+p}\right)\left(\mathrm{pr}_{2}^{2}\right)(x, y) \\
& =a\left(\left(T_{n}-T_{n+p}\right)\left(\mathrm{pr}_{1}^{2}\right)(x, y)+\left(T_{n}-T_{n+p}\right)\left(\mathrm{pr}_{2}^{2}\right)(x, y)\right),
\end{aligned}
$$

which ensures that the sequence $\left(T_{n}(\xi)(x, y)\right)_{n \in \mathbb{N}}$ is a Cauchy sequence. We get the same result if $T(\mathbf{1})(x, y)<0$ using the fact that $(x, y) \in X^{-}$and $(x, y) \in X_{n}^{-}$for every $n \in \mathbb{N}$. Finally, assume that $T(\mathbf{1})(x, y)=0$, that is $\lim _{n \rightarrow+\infty} T_{n}(\mathbf{1})(x, y)=0$; if $\xi$ is positive, we have $\xi \in \operatorname{co}(0,\|\xi\| \mathbf{1})$ and from Proposition 3.1, it follows $0 \leq T_{n}(\xi)(x, y) \leq\|\xi\| T_{n}(\mathbf{1})(x, y)$ for every $n \in \mathbb{N}$; hence we have $\lim _{n \rightarrow+\infty} T_{n}(\xi)(x, y)=0$. If $\xi$ is not positive, it is enough to apply the previous argument to the positive and negative part of $\xi$. Thus, we have shown that $\left(T_{n}(\xi)(x, y)\right)_{n \in \mathbb{N}}$ is a Cauchy sequence for every $(x, y) \in X$. A straightforward compact argument on $X$ yields that $\left(T_{n}(\xi)\right)_{n \in \mathbb{N}}$ is a Cauchy sequence in $C(X, \mathbb{R})$ and therefore it converges. Hence, we have obtained the existence of the limit operator $T: C(X, \mathbb{R}) \rightarrow C(X, \mathbb{R})$. If $(x, y) \in X^{+} \backslash X^{-}$, we have $T(\mathbf{1})(x, y)>0$ and the above argument shows that $T(\xi)(x, y) \geq 0$ for every positive $\xi \in C(X, \mathbb{R})$; analogously if $(x, y) \in X^{-} \backslash X^{+}$, we have $T(\mathbf{1})(x, y)<0$ and $T(\xi)(x, y) \leq 0$ for every positive $\xi \in C(X, \mathbb{R})$. If $(x, y) \in X^{+} \cap X^{-}$, we have $T(\mathbf{1})(x, y)=0$ and we have shown that in this case $T(\xi)(x, y)=0$ for every positive $\xi \in C(X, \mathbb{R})$. Hence, $T$ satisfies condition c) in Proposition 3.1.

A quantitative estimate of the convergence can be easily obtained by applying the same arguments in [10, (3)] (see also the proof of [15, Theorem 1.2]) to the cases $(x, y) \in X^{+}$and $(x, y) \in X^{-}$. We omit the details for the sake of brevity. As an immediate consequence of Theorem 4.9, we can state the following result concerning the convergence of sequences of vector-valued functions. As usual, we shall denote by $\mathbf{e}_{j}=\left(\delta_{i j}\right)_{i=1,2}, j=1,2$, the canonical basis of $\mathbb{R}^{2}$ and by $\langle\cdot, \cdot\rangle$ the scalar product in $\mathbb{R}^{2}$.

Theorem 4.10. Let $X$ be a convex compact subset of $\mathbb{R}^{2}$ and let $\left(T_{n}\right)_{n \in \mathbb{N}}$ be a sequence of continuous linear operators on $C\left(X, \mathbb{R}^{2}\right)$ satisfying condition (3.3) and assume that

(i) The sequence $\left(T_{n}\left(\mathrm{pr}_{i}^{2} \cdot \boldsymbol{e}_{j}\right)\right)_{n \in \mathbb{N}}, i=1,2$, converges to a vector-valued continuous function $h_{i} \in C\left(X, \mathbb{R}^{2}\right)$.

(ii) The sequence $\left(\left\|T_{n}(\varphi)(x, y)\right\|\right)_{n \in \mathbb{N}}$ is nonincreasing with respect to $n$ for any function $\varphi \in$ $C\left(X, \mathbb{R}^{2}\right)$ having convex components and $(x, y) \in X$.

Then, there exists a continuous linear operator $T: C\left(X, \mathbb{R}^{2}\right) \rightarrow C\left(X, \mathbb{R}^{2}\right)$ satisfying (3.3) and such that $\lim _{n \rightarrow+\infty} T_{n}(\varphi)(x, y)=T(\varphi)(x, y)$ for every $\varphi \in C\left(X, \mathbb{R}^{2}\right)$ uniformly with respect to $(x, y) \in X$.

Proof. The proof follows the same line of [10, Theorem 1.2]. For every $j, k=1,2$ and $n \in \mathbb{N}$, consider the operator $T_{j, k, n}: C(X, \mathbb{R}) \rightarrow C(X, \mathbb{R})$ defined by setting

$$
T_{j, k, n}(\xi)(x, y):=\left\langle T_{n}\left(\xi \cdot \mathbf{e}_{j}\right)(x, y), \mathbf{e}_{k}\right\rangle
$$

whenever $\xi \in C(X, \mathbb{R})$ and $(x, y) \in X$. Then, the sequence $\left(T_{j, k, n}\right)_{n \in \mathbb{N}}$ satisfies all assumptions of Theorem 4.9 and consequently if strongly converges to an operator $T_{j, k}: C(X, \mathbb{R}) \rightarrow C(X, \mathbb{R})$ satisfying (3.3). Now, consider the operator $T: C\left(X, \mathbb{R}^{2}\right) \rightarrow C\left(X, \mathbb{R}^{2}\right)$ defined by setting, for every $\varphi \in C\left(X, \mathbb{R}^{2}\right)$ and $(x, y) \in X$,

$$
T(\varphi)(x, y):=\left(T_{1,1}\left(\varphi_{1}\right)(x, y)+T_{2,1}\left(\varphi_{2}\right)(x, y), T_{1,2}\left(\varphi_{1}\right)(x, y)+T_{2,2}\left(\varphi_{2}\right)(x, y)\right),
$$

where $\varphi_{j}:=\left\langle\varphi, \mathbf{e}_{j}\right\rangle, j=1,2$, denotes the function $\varphi_{j}(x, y)=\left\langle\varphi(x, y), \mathbf{e}_{j}\right\rangle,(x, y) \in X$. Then, from (4.10), it follows that the sequence $\left(T_{n}\right)_{n \in \mathbb{N}}$ converges strongly to $T$ and this also implies that $T$ is convexity monotone; indeed (3.3) is satisfied by each $T_{n}$ and passing to the limit at every point $(x, y) \in X$ it is also satisfied by $T$. 
Finally, we state the corresponding result for set-valued continuous functions.

Theorem 4.11. Let $\left(L_{n}\right)_{n \in \mathbb{N}}$ be a sequence of continuous monotone linear operators on $C\left(X, \mathcal{K}\left(\mathbb{R}^{2}\right)\right)$ satisfying condition (3.4) and assume that

(i) The sequence $\left(L_{n}\left(\left\{\operatorname{pr}_{i}^{2} \cdot \boldsymbol{e}_{j}\right\}\right)_{n \in \mathbb{N}}, i, j=1,2\right.$, converges to a set-valued continuous function $h_{i, j} \in C\left(X, \mathbb{R}^{2}\right)$.

(ii) For every $j, k=1,2$, the sequence $\left(\left|\left\langle\eta_{n, j}(x, y), \boldsymbol{e}_{k}\right\rangle\right|\right)_{n \in \mathbb{N}}$ is nonincreasing whenever $\xi \in$ $C(X, \mathbb{R})$ is a convex function, $(x, y) \in X$ and $\eta_{n, j} \in C\left(X, \mathbb{R}^{2}\right)$ is such that $L_{n}\left(\left\{\xi \cdot \boldsymbol{e}_{j}\right\}\right)(x)=$ $\left\{\eta_{n, j}(x)\right\}$.

(ii) The sequence $\left(\left|T_{n}(\varphi)(x, y)\right|\right)_{n \in \mathbb{N}}$ is nonincreasing with respect to $n$ for any function $\varphi \in$ $C\left(X, \mathbb{R}^{2}\right)$ having convex components and $(x, y) \in X$.

Then, there exists a continuous monotone linear operator $L: C\left(X, \mathcal{K}\left(\mathbb{R}^{2}\right)\right) \rightarrow C\left(X, \mathcal{K}\left(\mathbb{R}^{2}\right)\right)$ such that $\lim _{n \rightarrow+\infty} L_{n}(f)(x, y)=L(f)(x, y)$ for every $f \in C\left(X, \mathcal{K}\left(\mathbb{R}^{2}\right)\right)$ uniformly with respect to $(x, y) \in X$.

Proof. Also in this case the proof follows the same line of [10, Theorem 2.4]. For every $n \in \mathbb{N}$, consider the operator $T_{n}:=T_{L_{n}}$ defined as in (4.8). From Lemma 4.2, the sequence $\left(T_{n}\right)_{n \in \mathbb{N}}$ satisfies the assumptions in Theorem 4.10 and therefore there exists a continuous linear operator $T: C\left(X, \mathbb{R}^{2}\right) \rightarrow C\left(X, \mathbb{R}^{2}\right)$ satisfying condition (3.3) and such that $\lim _{n \rightarrow+\infty} T_{n}(\varphi)=T(\varphi)$ for every $\varphi \in C\left(X, \mathbb{R}^{2}\right)$. From (4.9), we can define the continuous monotone linear operator $L:=L_{T}: C\left(X, \mathcal{K}\left(\mathbb{R}^{2}\right)\right) \rightarrow C\left(X, \mathcal{K}\left(\mathbb{R}^{2}\right)\right)$. First, we observe that the sequence $\left(L_{n}(\mathbf{1} \cdot \mathbf{B})\right)_{n \in \mathbb{N}}$ is equibounded. Indeed, $\mathbf{1} \cdot \mathbf{B} \subset \operatorname{co}\left(\delta_{k j} \mathbf{1} \cdot \mathbf{e}_{j} ; k, j=1,2\right)$ and since every $L_{n}$ is convexity monotone, we have $L_{n}(\mathbf{1} \cdot \mathbf{B}) \subset \operatorname{co}\left(\delta_{k j} L_{n}\left(\left\{\mathbf{1} \cdot \mathbf{e}_{j}\right\}\right), k, j=1,2\right.$; from assumption (i), the sequence $\left(L_{n}\left(\left\{\mathbf{1} \cdot \mathbf{e}_{j}\right\}\right)\right)_{n \in \mathbb{N}}$ converges to $\left\{h_{0, j}\right\}$ for every $j=1,2$ and therefore there exists $M>0$ such that $L_{n}(\mathbf{1} \cdot \mathbf{B}) \subset M \cdot \mathbf{B}$. Now, we show that for every $\varphi_{1}, \ldots, \varphi_{m} \in C\left([0,1], \mathbb{R}^{2}\right)$,

$$
\lim _{n \rightarrow+\infty} L_{n}\left(\operatorname{co}\left(\varphi_{1}, \ldots, \varphi_{m}\right)\right)=\operatorname{co}\left(L\left(\left\{\varphi_{1}\right\}\right), \ldots, L\left(\left\{\varphi_{m}\right\}\right)\right) .
$$

Indeed, for every $j=1, \ldots, m$ we know that $\lim _{n \rightarrow+\infty} L_{n}\left(\left\{\varphi_{j}\right\}\right)=L\left(\left\{\varphi_{j}\right\}\right)$ (i.e., for the associated operators, $\left.\lim _{n \rightarrow+\infty} T_{n}\left(\varphi_{j}\right)=T\left(\varphi_{j}\right)\right)$ and therefore, if $\varepsilon>0$, there exists $\nu \in \mathbb{N}$ such that $\left\|T_{n}\left(\varphi_{j}\right)-T\left(\varphi_{j}\right)\right\| \leq \varepsilon$ whenever $n \geq \nu$. It follows, for every $n \geq \nu$,

$$
\begin{aligned}
& \operatorname{co}\left(L_{n}\left(\left\{\varphi_{1}\right\}\right), \ldots, L_{n}\left(\left\{\varphi_{m}\right\}\right)\right) \subset \operatorname{co}\left(L\left(\left\{\varphi_{1}\right\}\right), \ldots, L\left(\left\{\varphi_{m}\right\}\right)\right)+\varepsilon \cdot \mathbf{B}, \\
& \operatorname{co}\left(L\left(\left\{\varphi_{1}\right\}\right), \ldots, L\left(\left\{\varphi_{m}\right\}\right)\right) \subset \operatorname{co}\left(L_{n}\left(\left\{\varphi_{1}\right\}\right), \ldots, L_{n}\left(\left\{\varphi_{m}\right\}\right)\right)+\varepsilon \cdot \mathbf{B} .
\end{aligned}
$$

Indeed, if $\varphi(x, y):=\sum_{j=1}^{m} \lambda_{j}(x, y) T_{n}\left(\varphi_{j}\right)(x, y)$ with $\lambda_{j}(x, y) \geq 0$ and $\sum_{j=1}^{m} \lambda_{j}(x, y)=1$, we can write

$$
\varphi(x, y):=\sum_{j=1}^{m} \lambda_{j}(x, y) T\left(\varphi_{j}\right)(x, y)+\sum_{j=1}^{m} \lambda_{j}(x, y)\left(T_{n}\left(\varphi_{j}\right)(x, y)-T\left(\varphi_{j}\right)(x, y)\right)
$$

and since $\left\|T_{n}\left(\varphi_{j}\right)(x, y)-T\left(\varphi_{j}\right)(x, y)\right\| \leq \varepsilon$, we obtain $\varphi \in \operatorname{co}\left(L\left(\left\{\varphi_{1}\right\}\right), \ldots, L\left(\left\{\varphi_{m}\right\}\right)\right)+\varepsilon \cdot \mathbf{B}$; the second inclusion in (4.12) is similar. From (4.12) and taking into account that every $L_{n}$ and $L$ are convexity monotone, (4.11) follows. Finally, let $f \in C\left(X, \mathcal{K}\left(\mathbb{R}^{2}\right)\right)$ and fix $\varepsilon>0$. From Lemma 4.1 and (4.6), there exist $\varphi_{1}, \ldots, \varphi_{m} \in \operatorname{Sel}(f)$ such that

$$
\operatorname{co}\left(\varphi_{1}, \ldots, \varphi_{m}\right) \leq f \leq \operatorname{co}\left(\varphi_{1}, \ldots, \varphi_{m}\right)+\varepsilon \cdot \mathbf{B} .
$$

Every $L_{n}$ is convexity monotone and therefore

$$
\operatorname{co}\left(L_{n}\left(\left\{\varphi_{1}\right\}\right), \ldots, L_{n}\left(\left\{\varphi_{m}\right\}\right)\right) \leq L_{n}(f) \leq \operatorname{co}\left(L_{n}\left(\left\{\varphi_{1}\right\}\right), \ldots, L_{n}\left(\left\{\varphi_{m}\right\}\right)\right)+\varepsilon M \cdot \mathbf{B} .
$$

Since $\varepsilon$ is arbitrarily chosen, from (4.11), we can conclude that $\lim _{n \rightarrow+\infty} L_{n}(f)=L(f)$. 
Many sequences of set-valued operators are obtained from their analogs in the single-valued setting. In this sense, the assumptions in Theorem 4.11 are quite natural since allow to obtain the convergence in the set-valued setting from the same assumptions in the single-valued setting.

\section{ACKNOWLEDGMENTS}

Work performed under the auspices of G.N.A.M.P.A. (INdAM).

\section{REFERENCES}

[1] F. Altomare, M. Campiti: Korovkin-type Approximation Theory and Its Applications, De Gruyter Studies in Mathematics 17, Berlin-Heidelberg-New York, (1994).

[2] F. Altomare, M. Cappelletti, V. Leonessa and I. Raşa: Markov Operators, Positive Semigroups and Approximation Processes, De Gruyter Studies in Mathematics 61, Berlin-Munich-Boston, (2015).

[3] H. Berens, G. G. Lorentz: Geometric theory of Korovkin sets, J. Approx. Theory, 15 (3) (1975), 161-189.

[4] M. Campiti: A Korovkin-type theorem for set-valued Hausdorff continuous functions, Le Mathematiche, 42 (I-II) (1987), 29-35.

[5] M. Campiti: Approximation of continuous set-valued functions in Fréchet spaces I, Rev. Anal. Numér. Théor. Approx., 20 (1-2) (1991), 15-23.

[6] M. Campiti: Approximation of continuous set-valued functions in Fréchet spaces II, Rev. Anal. Numér. Théor. Approx., 20 (1-2) (1991), 24-38.

[7] M. Campiti: Korovkin theorems for vector-valued continuous functions, in "Approximation Theory, Spline Functions and Applications" (Internat. Conf., Maratea, May 1991), 293-302, Nato Adv. Sci. Inst. Ser. C: Math. Phys. Sci. 356 , Kluwer Acad. Publ., Dordrecht, 1992.

[8] M. Campiti: Convergence of nets of monotone operators between cones of set-valued functions, Atti dell'Accademia delle Scienze di Torino, 126 (1992), 39-54.

[9] M. Campiti: Convexity-monotone operators in Korovkin theory, Rend. Circ. Mat. Palermo, 33 (1993), 229-238.

[10] M. Campiti: Korovkin-type approximation in spaces of vector-valued and set-valued functions, Applicable Analysis, 98 (13) (2019), 2486-2496.

[11] L. B. O. Ferguson, M. D. Rusk: Korovkin sets for an operator on a space of continuous functions, Pacific J. Math., 65 (2) (1976), 337-345.

[12] W. Heping: Korovkin-type theorem and application, J. Approx. Theory, 132 (2005), 258-264.

[13] K. Keimel, W. Roth: A Korovkin type approximation theorem for set-valued functions, Proc. Amer. Math. Soc., 104 (1988), 819-824.

[14] K. Keimel, W. Roth: Ordered cones and approximation, Lecture Notes in Mathematics, 1517, Springer-Verlag Berlin Heidelberg, (1992).

[15] N. I. Mahmudov: Korovkin-type theorems and applications, Cent. Eur. J. Math., 7 (2) (2009), 348-356.

[16] T. Nishishiraho: Convergence of quasi-positive linear operators, Atti Sem. Mat. Fis. Univ. Modena, 29 (1991), 367-374.

Michele CAMPITI

UNIVERSITY OF SALENTO

Department of Mathematics and Physics "E. De Giorgi"

VIA PER ARNESANO, 73100, LECCE, ITALY

ORCID: 0000-0003-3794-1878

E-mail address: michele.campiti@unisalento.it 